\title{
ACEITAÇÃO DE SOBREMESAS LÁCTEAS DIETÉTICAS E FORMULADAS COM AÇÚCAR: TESTE AFETIVO E MAPA DE PREFERÊNCIA INTERNO ${ }^{1}$
}

\author{
Ana Paula Vital de OLIVEIRA², Karina FRASSON², Tereza Cristina Avancini de ALMEIDA²,
} Marta de Toledo BENASSI ${ }^{2, *}$

\section{RESUMO}

A aceitação de sobremesas lácteas de chocolate (três pudins com açúcar, cinco pudins dietéticos e dois flans comerciais) foi avaliada por 56 consumidores utilizando delineamento de blocos completos balanceados e empregando-se uma escala hedônica estruturada de nove pontos. Os provadores foram caracterizados através de um questionário para coleta de informações pessoais e hábitos de consumo de produtos dietéticos e pudins. Os dados de aceitação foram analisados por ANOVA e teste de médias. Empregou-se, também, a metodologia de Mapa de Preferência Interno utilizando diferentes técnicas estatísticas (Análise de Componentes Principais e Escala Multidimensional) associadas à Análise de Agrupamentos, para segmentação dos consumidores. Considerando-se as notas médias, observou-se que os pudins dietéticos se destacaram pela boa aceitação. O emprego da técnica de Escala Multidimensional proporcionou uma avaliação mais abrangente do comportamento dos provadores do que a Análise de Componentes Principais, permitindo identificar dois grandes grupos de consumidores: os que preferiam os produtos (pudins e flan) de uma determinada marca e os que preferiam os pudins dietéticos. Os resultados demonstram a eficiência do emprego do mapa na identificação e caracterização de preferências e de grupos de consumidores.

Palavras-chave: pudim; flan; Escala Multidimensional; Análise de Componentes Principais; chocolate.

\section{SUMMARY}

ACCEPTABILITY OF SWEETENED AND LOW CALORIE DAIRY DESSERTS: AFFECTIVE TESTS AND INTERNAL PREFERENCE MAPPING. The acceptance of commercial chocolate dairy desserts (three regular puddings, five dietetic puddings and two regular flans) was evaluated by 56 consumers in a balanced-block design, using a nine-point structured hedonic scale. Panelists were characterized using a questionnaire asking for personal information and consumer's behavior towards dietary products and puddings consumption. The acceptance data were analyzed by ANOVA with means comparison and using the technique of Internal Preference Mapping (MDPREF) applying different statistical methods (Principal Components Analysis and Muldimensional Scale) associated to Cluster Analysis to provide consumers segmentation. Considering the mean values, it was emphasized the good acceptance of dietetic puddings. The application of the Muldimensional Scale technique provided a more comprehensive evaluation of the panelists behavior than the Principal Components Analysis, allowing to identify two main groups of consumers: the ones that preferred the products of a specific brand (puddings and flan) and the ones that preferred the dietetic puddings. Results demonstrate the efficiency of the use of mapping techniques in the identification and characterization of preferences and consumers' groups.

Keywords: pudding; flan; Multidimensional Scale; Principal Components Analysis; chocolate.

\section{1 - INTRODUÇÃO}

A partir dos anos 80, os dietéticos vêm revolucionando o mercado nacional, criando uma verdadeira "onda diet" [4]. Segundo a Associação Brasileira de Produtos Dietéticos, o setor "light", em geral, cresceu em torno de 14 vezes na década, passando de US\$160 milhões em 90 para US\$2,2 bilhões em 2000, com uma estimativa de US\$7 bilhões para 2005. Nesses dez anos, surgiram 750 versões de alimentos com baixas calorias ou isenção total de açúcar; os segmentos que mais se destacam foram os de refrigerantes, sorvetes, iogurtes e sobremesas [10].

Os produtos elaborados com sacarose geralmente se destacam pela aparência e sabor. Além do gosto doce, a sacarose aumenta a viscosidade do meio, conferindo textura adequada e estabilidade. A utilização de edulcorante pode ser adequada em relação ao sabor, mas, muitas vezes, não proporciona as características de

\footnotetext{
Recebido para publicação em 25/04/2003. Aceito para publicação em 23/08/2004 (001116).

2. Depto. Tecnologia de Alimentos e Medicamentos/UEL, Caixa Postal 6001, CEP: 86051-970, Londrina/PR. E-mail: martatb@uel.br

3. E-mail: sensory@uol.com.br

* A quem a correspondência deve ser enviada.
}

textura e aparência desejadas [1]. Os indivíduos que precisam substituir o açúcar por adoçantes não calóricos, procuram produtos que sejam dotados de características sensoriais próximas as de alimentos formulados com sacarose [5].

Nos últimos anos, vem crescendo o interesse, na área de análise sensorial, por estudos de consumidor. Os resultados de testes afetivos são, tradicionalmente, avaliados por análise de variância univariada e teste de médias. Assim, às vezes, a simples média de aceitação, quando existem categorias de consumidores com preferências opostas, faz com que o resultado de um grupo cancele o do outro [3, 8, 14].

A técnica de Mapa de Preferência utiliza análise estatística multivariada para obter, num espaço multidimensional, uma representação gráfica das diferenças de aceitação entre produtos, identificando o indivíduo e suas preferências $[9,20]$. Permite, ainda, identificar as amostras mais aceitas pela maioria da população do estudo e, ao considerar a individualidade de cada provador, caracterizar grupos com diferentes preferências e padrões de consumo. Os Mapas podem ser divididos em duas categorias: interno, quando se constrói o espaço vetorial sobre dados de aceitação/preferência gerados a partir de testes afetivos, e externo, onde o espaço vetorial é construído com dados de análise descritiva ou outras 
caracterizações físico-químicas e, depois correlacionado com dados de aceitação [12, 13].

O Mapa de Preferência Interno (MDPREF) é gerado a partir de uma matriz com os dados de aceitação de uma equipe de consumidores, sobre um mesmo conjunto de amostras [13], não permitindo o uso de delineamento de blocos incompletos [8].

A maioria dos autores que trabalha com o MDPREF utiliza a Análise de Componentes Principais (ACP) para avaliar a matriz de dados de aceitação. Considera-se que os indivíduos da ACP são os consumidores e, as variáveis, são as amostras, identificando a maior variação dentro dos dados de preferência para a extração dos componentes [20]. Essa técnica promove a redução da dimensionalidade do conjunto original de variáveis, com a menor perda de informação possível, permitindo o agrupamento, em gráficos no espaço bi ou tridimensional, de indivíduos que tenham comportamento análogo com relação às variáveis [6]. Alguns programas/ pacotes específicos da área, "Senstools" [18] e "PC-MDS Multidimensional Statistic Package" [21], no entanto, trabalham com outra técnica estatística multivariada, a Escala Multidimensional (EM). A EM está baseada num modelo vetorial, em que o conjunto de estímulos (produtos), é embutido em um espaço, onde cada indivíduo é representado por um vetor. O resultado consiste em um conjunto de dimensões de preferência, representando as diferenças entre as amostras, e um conjunto de vetores, um para cada provador, que mostram a direção individual de preferência [13].

Alguns autores têm reportado, ainda, que a segmentação dos consumidores, realizada por uma Análise de Agrupamentos (AA), associada ao MDPREF poderia facilitar a discussão dos resultados de preferência [2, 23].

O objetivo do trabalho foi verificar a eficiência de diferentes técnicas multivariadas (Análise de Componentes Principais e Escala Multidimensional) na elaboração de um Mapa de Preferência Interno, para avaliação da aceitação de dez produtos comerciais, pudins e flans formulados com açúcar e pudins dietéticos.

\section{2 - MATERIAL E MÉTODOS}

\section{1 - Material}

Foram utilizadas sobremesas lácteas comerciais de chocolate disponíveis no mercado: flans (marcas A e $\mathrm{B}$ ), pudins com açúcar (marcas A, B e C) e pudins dietéticos (marcas A, B, C, D, E, F). Para designar as amostras serão utilizadas as letras $\mathrm{F}$ (flan com açúcar), $\mathrm{P}$ (pudim com açúcar) e D (pudim dietético), seguidas de letras maiúsculas (A, B, C, D, E, F) em subscrito para indicar as marcas. A descrição de cada produto, de acordo com o seu rótulo, pode ser observada na Tabela 1 . As misturas em pó foram dissolvidas em $500 \mathrm{~mL}$ de leite desnatado e aquecidas de acordo com as instruções do fabricante. Foram, então, acondicionadas (em torno de $40 \mathrm{~g}$ ) em copos plásticos de $100 \mathrm{~mL}$, transparentes e com tampa. Após resfriamento a temperatura ambiente, as amostras foram mantidas sob refrigeração a $7 \pm 2{ }^{\circ} \mathrm{C}$ por 24 horas até a análise sensorial.

TABELA 1. Descrição dos pós para preparo de pudim de chocolate comerciais.

\begin{tabular}{|c|c|}
\hline Tipo* & Ingredientes/Aditivos \\
\hline $\mathrm{F}_{\mathrm{A}}$ & Açúcar, amido, cacau em pó, sal, aroma imitação, gomas carragena e jataí \\
\hline $\mathrm{F}_{\mathrm{B}}$ & Açúcar, amido, cacau em pó, sal, aromatizante, gomas carragena e jataí \\
\hline $\mathrm{P}_{\mathrm{A}}$ & Açúcar, amido, cacau em pó, sal, aromatizante \\
\hline $\mathrm{P}_{\mathrm{B}}$ & Açúcar, amido, cacau em pó, sal, aromatizante \\
\hline $\mathrm{P}_{\mathrm{C}}$ & Açúcar, amido, cacau em pó, corante e aroma artificial \\
\hline $\mathrm{D}_{\mathrm{A}}$ & Aspartame, amido, cacau em pó, aroma imitação, gomas carragena e jataí \\
\hline $\mathrm{D}_{\mathrm{B}}$ & Aspartame, manitol, amido, cacau em pó, aroma imitação, gomas carragena e jataí \\
\hline $\mathrm{D}_{\mathrm{D}}$ & $\begin{array}{l}\text { Ciclamato, sacarina, amido, cacau, sal, aromatizantes, corantes (tartrazina, } \\
\text { vermelho bordeaux, indigotina), corante caramelo, gomas carragena e jataí }\end{array}$ \\
\hline$D_{E}$ & $\begin{array}{l}\text { Aspartame, acesulfame } \mathrm{K} \text {, amido, cacau em pó, sal, aroma natural reforçado de } \\
\text { baunilha, gomas carragena e jataí, conservante ácido sórbico }\end{array}$ \\
\hline$D_{F}$ & $\begin{array}{l}\text { Ciclamato, sacarina, amido, cacau, sal, aromatizantes chocolate, doce de leite e } \\
\text { leite condensado, corante caramelo, goma carragena }\end{array}$ \\
\hline
\end{tabular}

\section{2 - Teste de aceitação}

Para caracterização da equipe, foi entregue um questionário de coleta de dados de faixa etária, sexo, escolaridade e hábitos de consumo referentes ao produto em questão e produtos dietéticos e/ou adoçantes em geral.

Os testes foram realizados em laboratório de Análise Sensorial, empregando-se cabines individuais e luz branca. As amostras foram servidas a temperatura de refrigeração em recipiente codificado com três dígitos, uma por vez, de forma seqüencial. Entre as provas foi oferecida água.

Foram utilizados cinqüenta e seis provadores, não selecionados, de idades variáveis, alunos, professores e funcionários da universidade. Empregou-se delineamento de blocos completos e a ordem de apresentação das amostras foi aleatorizada dentro da sessão. Cada provador avaliou duas amostras, na $1^{\text {a }}$ e $3^{\text {a }}$ sessões, e três amostras, na $2^{\underline{a}}$ e $4^{\text {a }}$ sessões.

Utilizou-se uma escala estruturada de nove pontos ancorada, com termos verbais, nos extremos e no meio. Solicitou-se, ainda, que fossem citadas as características mais e menos apreciadas (Figura 1).

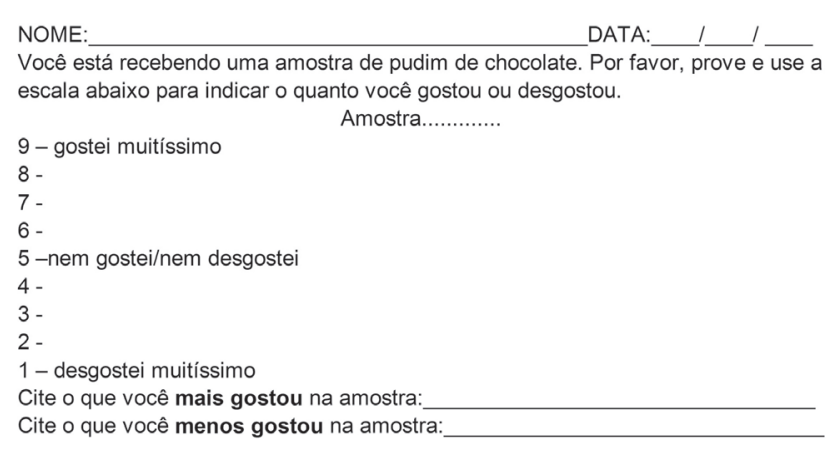

FIGURA 1. Ficha utilizada para teste de aceitação dos produtos. 


\section{3 - Análise estatística}

Os resultados de aceitação foram submetidos à análise de variância (ANOVA), considerando-se como causas de variação amostra, provador e sessão, e teste de médias (Tukey, $\mathrm{p} \leq 0,05$ ) [19]. Com base nas informações dos consumidores e nos resultados do teste afetivo, foram construídos histogramas.

Com a finalidade de analisar os dados afetivos levando-se em consideração a resposta individual de cada consumidor e não somente a média do grupo, os dados de aceitação foram, também, analisados pela metodologia do Mapa de Preferência Interno e Análise de Agrupamentos (AA). O mapa foi gerado empregando-se diferentes técnicas: Escala Multidimensional [18] e Análise de Componentes Principais [22]. No caso da EM, o pacote estatístico empregado [18] realiza automaticamente a AA. Para o mapa gerado por ACP, a AA foi realizada em separado utilizando-se a opção de Dendrograma no programa STATISTICA [22].

\section{3 - RESULTADOS E DISCUSSÃO}

\section{1 - Caracterização dos provadores}

Foram empregados 56 provadores, 22 homens e 34 mulheres, dos quais $71 \%$ eram alunos de graduação e pós-graduação e 39\%, funcionários e professores. A maioria dos provadores era jovem, $71 \%$ estavam na faixa até 35 anos, e mais da metade (52\%) da população estudada possuía nivel de escolaridade igual ou superior ao $3^{\circ}$ grau.

Praticamente todos os entrevistados (98\%) relataram que gostavam do sabor chocolate. Com relação à freqüência de consumo, 59\% consumia moderadamente sobremesas lácteas mas não estavam habituados a consumir o produto industrializado na forma de pó para preparo: 66\% empregavam ocasionalmente e $20 \%$ nunca haviam consumido. Da equipe utilizada, apenas $27 \%$ consumiam freqüentemente alimentos dietéticos e/ou adoçantes e um terço dos consumidores nunca haviam consumido esse tipo de produto (Figura 2).
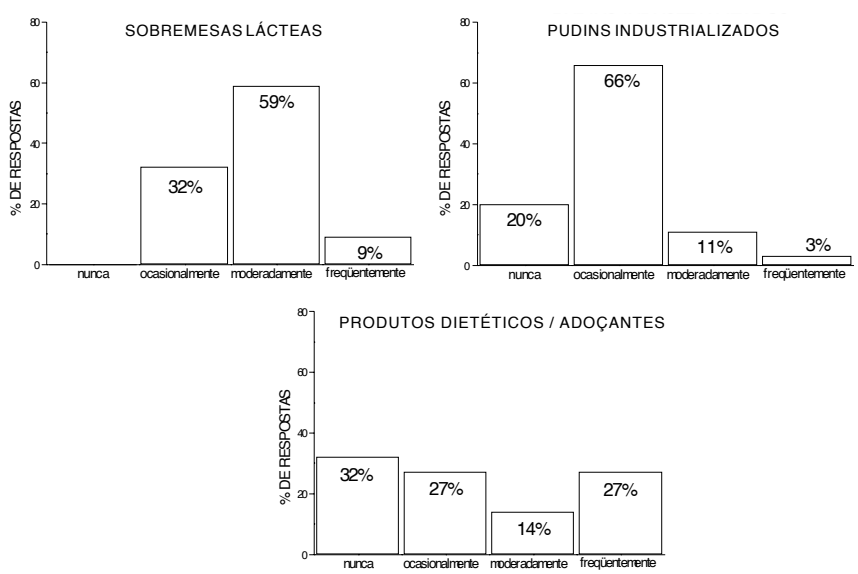

FIGURA 2. Freqüência de consumo dos produtos reportada pelos provadores (ocasionalmente, algumas vezes por ano; moderadamente, algumas vezes por mês; freqüentemente, algumas vezes na semana).

\section{2 - Avaliação da aceitação das amostras}

A Tabela 2 apresenta a nota média de aceitação das dez amostras e as porcentagens de aprovação, indiferença e rejeição, das amostras separadas por classes de produtos (flans, pudins com açúcar e dietéticos).

Para pudins com açúcar foi observada heterogeneidade nas médias de aceitação $(5,5$ a 6,9$)$ e porcentagem de aprovação (52 a 86\%) das diversas marcas. Os flans obtiveram uma aceitação intermediária (médias de 5,8 e 6,5), no entanto um dos produtos $\left(\mathrm{F}_{\mathrm{A}}\right)$ obteve o dobro da rejeição do outro (Tabela 2).

Os pudins dietéticos se destacaram pela boa aceitação, não havendo diferença significativa na nota média de aceitação $(6,7$ a 7,0)(Tabela 2). Observou-se que os pudins que obtiveram as maiores médias e porcentagens de aceitação $\left(D_{A}, D_{B}\right.$ e $\left.D_{E}\right)$ empregavam aspartame (sozinho ou associado a manitol e acesulfame) e, os de menor média e maior porcentagem de indiferença e rejeição $\left(D_{D}\right.$ e $\left.D_{F}\right)$, utilizavam como edulcorantes sacarina e ciclamato. A aceitação foi maior que a descrita por IOP, BELEIA \& SILVA [1 1] para pudins de chocolate formulados com diferentes combinações de ciclamato, sacarina e esteviosídeo (médias de 3,9 a 6,2) e avaliados por 24 provadores que consumiam com regularidade produtos dietéticos.

TABELA 2. Nota média da aceitação e porcentagens de aprovação, indiferença e rejeição dos produtos.

\begin{tabular}{ccccc}
\hline Tipo $^{*}$ & Nota $^{\star \star}$ & \% de aprovação & \% de indiferença & \% de rejeição $^{\star \star \star}$ \\
\hline$F_{A}$ & $5,8^{\mathrm{Dc}}$ & 63 & 9 & 28 \\
$\mathrm{~F}_{\mathrm{B}}$ & $6,5^{\mathrm{ab}}$ & 77 & 9 & 14 \\
\hline $\mathrm{P}_{\mathrm{A}}$ & $6,1^{\mathrm{abc}}$ & 64 & 18 & 18 \\
$\mathrm{P}_{\mathrm{B}}$ & $6,9^{\mathrm{a}}$ & 86 & 5 & 9 \\
$\mathrm{PC}_{\mathrm{C}}$ & $5,5^{\mathrm{c}}$ & 52 & 20 & 28 \\
\hline $\mathrm{D}_{\mathrm{A}}$ & $6,9^{\mathrm{a}}$ & 86 & 9 & 5 \\
$\mathrm{D}_{\mathrm{B}}$ & $6,8^{\mathrm{a}}$ & 82 & 11 & 7 \\
$\mathrm{D}_{\mathrm{D}}$ & $6,7^{\mathrm{ab}}$ & 77 & 14 & 9 \\
$\mathrm{D}_{\mathrm{E}}$ & $7,0^{\mathrm{a}}$ & 86 & 3 & 11 \\
$\mathrm{D}_{\mathrm{F}}$ & $6,7^{\mathrm{ab}}$ & 77 & 7 & 16 \\
\hline
\end{tabular}

* F= flan com açúcar; $\mathrm{P}=$ pudim com açúcar; $\mathrm{D}=$ pudim dietético. Letras maiúsculas em subscrito (A, B, C, D, E, F) indicam a marca dos produtos.
$* *$ Média de 56 provadores, letras diferentes na mesma coluna indicam diferença significativa $(\mathrm{p} \leq 0,05)$.

***\% de aprovação= porcentagem de notas de 6 a 9; \% de indiferença= porcentagem de notas 5; \% de rejeição= porcentagem de notas de 1 a 4 .

Com relação às marcas, observou-se para a marca A diferença de aceitação entre flan e pudins com açúcar e dietético, com rejeição variando de 5 a $28 \%$. No caso da marca $\mathrm{B}$, todos os produtos obtiveram um alto índice de aceitação (entre 77 e 86\%) (Tabela 2). Para as três classes de produtos dessa marca, a cor acentuada de chocolate foi citada pelos consumidores como uma das características mais apreciadas (em torno de 30\% das respostas). Em estudo empregando análise instrumental de cor observou-se que os produtos da marca $\mathrm{B}$, independentemente da classe, se diferenciaram dos outros de mercado mostrando-se mais escuros e avermelhados [17]. CHAN \& MARTINELLI [7] observaram, trabalhando com 54 adultos em diferentes faixas 
etárias (20 a 35 anos e 60 a 90 anos), que o incremento na intensidade de cor marrom aumentou a aceitação de pudins de chocolate.

O fato da maioria dos provadores nunca e/ou ocasionalmente haver consumido alimentos dietéticos e/ ou adoçantes não foi um obstáculo para que percebessem o gosto residual. Esse atributo foi citado como característica negativa para todos os pudins dietéticos, com variação na porcentagem de respostas de 5 a 30\% dependendo do produto. Não se observou diferença significativa $(p \geq 0,05)$ nas médias de aceitação dos produtos comparando-se consumidores com diferentes niveis de familiaridade com dietéticos (nunca, ocasionalmente, moderadamente ou freqüentemente consumiam).

As três amostras que tiveram maiores notas médias foram de dois pudins dietéticos $\left(D_{E}\right.$ e $\left.D_{A}\right)$ e um pudim com açúcar $\left(\mathrm{P}_{\mathrm{B}}\right)$, com médias de 7,0,6,9 e 6,9, e porcentagem de aprovação de $86 \%$ (Tabela 2 ). Verificou-se que a textura, sabor e cor foram os atributos que mais influenciaram os consumidores com relação à aceitação das duas amostras (Tabela 3).

TABELA 3. Características sensoriais mais apreciadas e menos apreciadas citadas pelos provadores em relação às amostras que tiveram maiores e menores porcentagens de aprovação.

\begin{tabular}{|c|c|c|c|c|c|}
\hline Tipo* & & $\begin{array}{l}\text { Características } \\
\text { mais apreciadas }\end{array}$ & $\begin{array}{c}\% \text { de } \\
\text { respostas }\end{array}$ & $\begin{array}{c}\text { Características } \\
\text { menos apreciadas }\end{array}$ & $\begin{array}{c}\% \text { de } \\
\text { respostas }\end{array}$ \\
\hline \multirow{9}{*}{$\begin{array}{c}\text { Maior } \\
\text { porcentagem } \\
\text { de aprovação }\end{array}$} & \multirow{3}{*}{$D_{E}$} & Textura & 43 & Gosto residual & 18 \\
\hline & & Sabor & 34 & Cor & 13 \\
\hline & & Cor & 27 & Sabor & 11 \\
\hline & \multirow{3}{*}{$\mathrm{D}_{\mathrm{A}}$} & Sabor & 38 & Pouca doçura & 23 \\
\hline & & Textura & 34 & Sabor & 14 \\
\hline & & Cor & 13 & Gosto residual & 5 \\
\hline & \multirow{3}{*}{$\mathrm{P}_{\mathrm{B}}$} & Sabor & 39 & Textura & 18 \\
\hline & & Textura & 29 & Cor & 13 \\
\hline & & Cor & 29 & Sabor & 9 \\
\hline \multirow{6}{*}{$\begin{array}{c}\text { Menor } \\
\text { porcentagem } \\
\text { de aprovação }\end{array}$} & \multirow{3}{*}{$\mathrm{F}_{\mathrm{A}}$} & Textura & 27 & Textura & 40 \\
\hline & & Cor & 25 & Doçura & 20 \\
\hline & & Sabor & 21 & Sabor & 13 \\
\hline & \multirow{3}{*}{$\mathrm{Pc}$} & Sabor & 20 & Sabor & 29 \\
\hline & & Textura & 16 & Textura & 29 \\
\hline & & Cor & 11 & Cor & 23 \\
\hline
\end{tabular}

${ }^{\mathrm{F}} \mathrm{F}$ flan com acúcar; $\mathrm{P}=$ pudim com açúcar; $\mathrm{D}=$ pudim dietético. Letras maiúsculas em subscrito (A,B,C,E) indicam a marca dos produtos.

Apesar de não haver diferença significativa na aceitação, pode-se observar que a distribuição de notas é distinta (Figura 3). $\mathrm{O}_{\mathrm{B}}$ e o $\mathrm{D}_{\mathrm{E}}$ receberam um maior número de notas 8 e 9 e obtiveram menores porcentagens de indiferença (3\% e 5\%) comparadas ao $\mathrm{D}_{\mathrm{A}}(9 \%)$. No entanto, a rejeição do $\mathrm{D}_{\mathrm{E}}(11 \%)$ foi o dobro da obtida pelo $\mathrm{D}_{\mathrm{A}}(5 \%)$, podendo estar associada aos índices de percepção do gosto residual (18 e 5\%, para $\mathrm{D}_{\mathrm{E}}$ e $\mathrm{D}_{\mathrm{A}}$ respectivamente). A rejeição do $\mathrm{P}_{B}$ foi intermediária (9\%). $\mathrm{O} \mathrm{D}_{\mathrm{A}}$ apresentou uma distribuição próxima ao normal; para $\mathrm{D}_{\mathrm{E}}$ e $\mathrm{P}_{\mathrm{B}}$ observou-se, que apesar da boa aceitação, haviam grupos de consumidores que rejeitavam as amostras.
As amostras que receberam as menores notas médias $(5,5$ e 5,8$)$ foram correspondentes a um pudim formulado com açúcar $\left(\mathrm{P}_{\mathrm{C}}\right)$ e um flan $\left(\mathrm{F}_{\mathrm{A}}\right)$. Esses produtos diferiram significativamente das 4 amostras mais apreciadas, e obtiveram os menores índices de aprovação (52\% e 63\%, respectivamente) e os maiores de rejeição (28\%) (Tabela 2). O pudim foi rejeitado tanto pelas características de sabor e textura ( $29 \%$ cada), quanto pela cor (23\%). Para o flan, a textura foi decisiva para a baixa aceitação, sendo citada como o atributo menos apreciado por $40 \%$ dos provadores (Tabela 3 ).
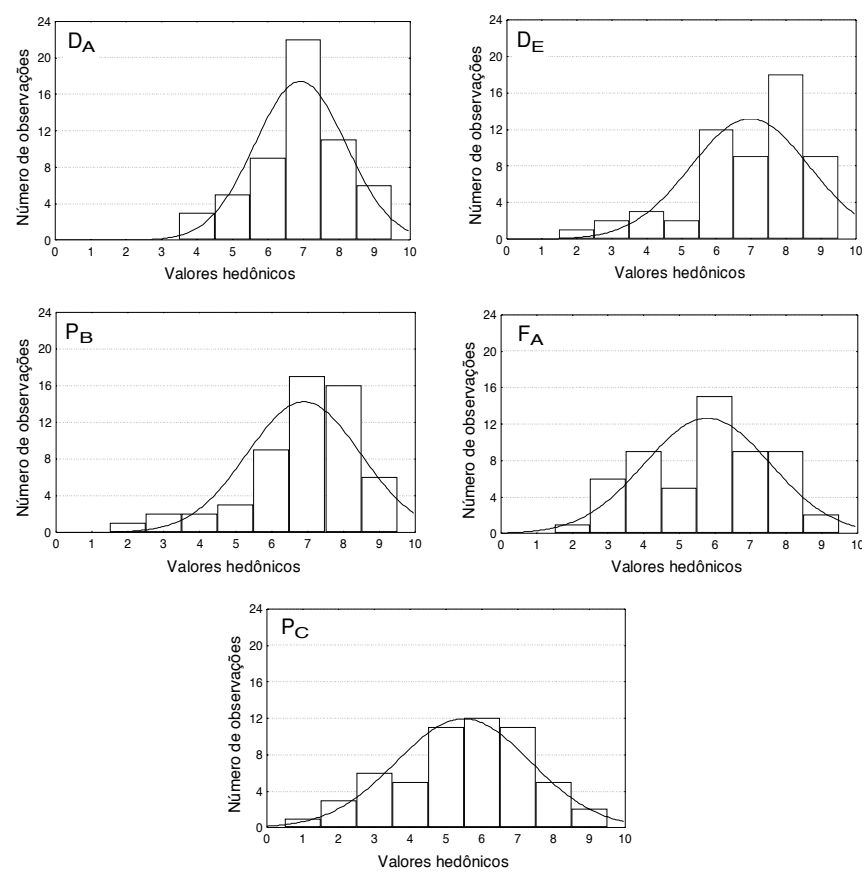

FIGURA 3. Histograma de freqüência dos valores hedônicos atribuídos às amostras* que tiveram maiores e menores porcentagens de aprovação. ( 1 = desgostei muitíssimo, 5 = nem gostei/nem desgostei, 9 = gostei muitíssimo) *F= flan com açúcar; $\mathrm{P}=$ pudim com açúcar; $\mathrm{D}=$ pudim dietético. Letras maiúsculas em subscrito (A, B, C, E) indicam a marca dos produtos.

Em trabalho anterior, empregando-se análise de textura instrumental, observou-se que havia diferença significativa entre os produtos com menores índices de aprovação, que apresentavam valores muito altos $\left(\mathrm{F}_{\mathrm{A}}\right)$ ou baixos $\left(\mathrm{P}_{\mathrm{C}}\right)$ de dureza, e os mais apreciados, que tinham um perfil intermediário [16]. A avaliação instrumental de cor mostrou que o $\mathrm{P}_{\mathrm{C}}$ se diferenciava por apresentar os maiores valores de luminosidade e tonalidade cromática, indicando uma cor mais clara e amarelada [17].

Com relação aos histogramas dos produtos com menores índices de aprovação (Figura 3), apesar das duas amostras terem apresentado uma aceitação próxima a nota 5 (nem gostei/nem desgostei), observou-se perfis diferenciados. $\mathrm{O}_{\mathrm{C}}$ apresentou uma distribuição próxima a normal, com uma alta proporção de indiferença (20\%). O F $\mathrm{A}_{\mathrm{A}}$ recebeu a mesma porcentagem de notas na região de rejeição ao produto (28\%), mas teve uma indi- 
ferença bem menor (9\%). Esse tipo de comportamento se aproxima de uma distribuição sigmoidal dupla, em que o histograma tem dois picos (regiões de aprovação e rejeição) separados por um vale (região de indiferença). Nesse caso, o emprego da média sugeriu indiferença ao produto e não permitiu observar a segmentação dos consumidores, que se dividiam em um grupo que aprovava o produto e outro que o rejeitava.

\section{3 - Mapa de preferência interno}

\subsection{1 - Escala Multidimensional}

Utilizando a técnica de Escala Multidimensional, o Mapa de Preferência foi gerado por meio da primeira e segunda dimensões, que explicaram em conjunto, 39\% da variabilidade dos provadores com relação à aceitação das amostras. A Figura 4 mostra a localização dos consumidores dentro do mesmo espaço vetorial gerado para as amostras. A Dimensão I pode ser considerada como a de preferência: amostras mais preferidas foram alocadas à direita e as menos preferidas mais à esquerda.

No gráfico, os provadores foram representados por pontos no espaço vetorial, que indicavam a direção de preferência de cada consumidor pelo conjunto de produtos, ou seja, os consumidores ficaram localizados próximos à região das amostras que eles preferiram. Os pontos correspondentes a cada indivíduo foram alocados em um círculo e a preferência individual pode ser obtida traçando-se um vetor passando pela origem até esse ponto e traçando-se linhas perpendiculares de cada estímulo (amostra) até o vetor. A direção do vetor representa a direção de aumento pessoal de "preferência” para um consumidor, assim, as amostras menos apreciadas ou menos preferidas são projetadas mais longe do indivíduo [13]. A seqüência de preferência dos vetores não representa exatamente as notas de cada indivíduo, mas são projeções sobre a dimensão de preferência que apresentam o melhor ajuste para os dados originais [15].

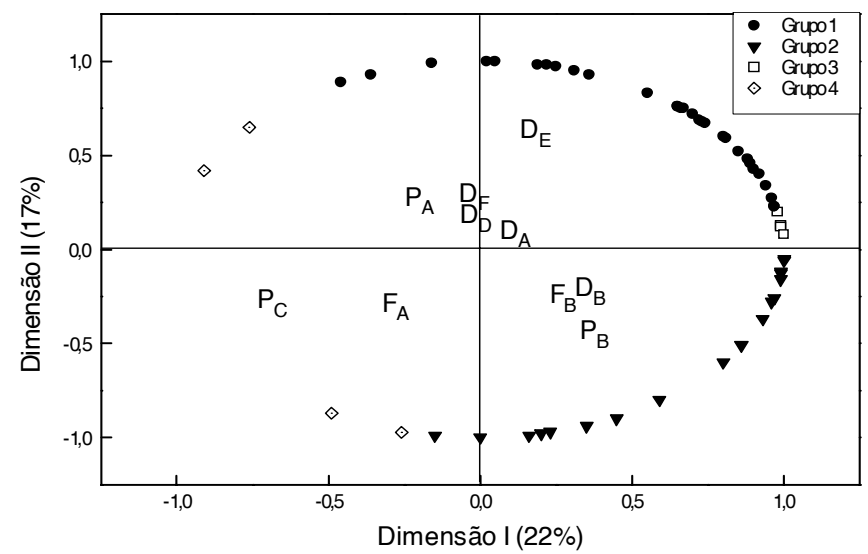

FIGURA 4. Projeção do parâmetro de aceitação para Dimensões I e II empregando-se Escala Muldimensional: configuração das amostras* e consumidores.

* $\mathrm{F}=$ flan com açúcar; $\mathrm{P}=$ pudim com açúcar; $\mathrm{D}=$ pudim dietético. Letras maiúsculas em subscrito (A,B,C,D,E,F) indicam a marca dos produtos.
No programa utilizado (SENSTOOLS) [18], a EM é, ainda, seguida automaticamente por uma segmentação dos consumidores realizada por uma Análise de Agrupamentos, que permitiu a identificação de quatro grupos. A Tabela 4 mostra as notas médias recebidas pelas amostras nos diferentes grupos.

Observou-se que a maioria dos consumidores (54\%, grupo 1) ficou localizada na região superior direita do mapa, onde se situavam duas das amostras com maiores notas médias e havia predominância de pudins dietéticos. Os provadores do grupo gostaram especialmente do $D_{E}$, que foi a amostra que teve maior média e gostaram menos dos flans e das amostras da marca $\mathrm{B}$ e $\mathrm{P}_{\mathrm{C}}$ (Figura 4 e Tabela 4).

TABELA 4. Notas médias de aceitação das amostras considerando-se toda a equipe e cada grupo*.

\begin{tabular}{cccccc}
\hline $\begin{array}{c}\text { Provadores/ } \\
\text { amostras }\end{array}$ & Equipe & Grupo 1 & Grupo 2 & Grupo 3 & Grupo 4 \\
\hline $\mathrm{N}^{\circ}$ total & 56 & 30 & 18 & 4 & 4 \\
\hline $\mathrm{F}_{\mathrm{A}}$ & 5,8 & $5,5^{\mathrm{cd}}$ & $6,0^{\mathrm{bcd}}$ & $5,3^{\mathrm{ab}}$ & $7,3^{\mathrm{a}}$ \\
$\mathrm{F}_{\mathrm{B}}$ & 6,5 & $6,3^{\mathrm{bcd}}$ & $6,9^{\mathrm{bc}}$ & $6,5^{\mathrm{ab}}$ & $6,0^{\mathrm{a}}$ \\
\hline $\mathrm{P}_{\mathrm{A}}$ & 6,1 & $6,6^{\mathrm{abc}}$ & $5,2^{\mathrm{d}}$ & $5,8^{\mathrm{ab}}$ & $7,0^{\mathrm{a}}$ \\
$\mathrm{P}_{\mathrm{B}}$ & 6,9 & $6,4^{\mathrm{acd}}$ & $7,7^{\mathrm{a}}$ & $7,5^{\mathrm{a}}$ & $6,5^{\mathrm{a}}$ \\
$\mathrm{P}_{\mathrm{C}}$ & 5,5 & $5,2^{\mathrm{d}}$ & $5,7^{\mathrm{cd}}$ & $4,0^{\mathrm{b}}$ & $8,0^{\mathrm{a}}$ \\
\hline $\mathrm{D}_{\mathrm{A}}$ & 6,9 & $7,0^{\mathrm{ab}}$ & $6,7^{\mathrm{bcd}}$ & $6,0^{\mathrm{ab}}$ & $8,3^{\mathrm{a}}$ \\
$\mathrm{D}_{\mathrm{B}}$ & 6,8 & $6,4^{\mathrm{bcd}}$ & $7,4^{\mathrm{ab}}$ & $7,0^{\mathrm{ab}}$ & $6,0^{\mathrm{a}}$ \\
$\mathrm{D}_{\mathrm{D}}$ & 6,7 & $6,9^{\mathrm{ab}}$ & $6,1^{\mathrm{bcd}}$ & $6,8^{\mathrm{ab}}$ & $7,8^{\mathrm{a}}$ \\
$\mathrm{D}_{\mathrm{E}}$ & 7,0 & $7,7^{\mathrm{a}}$ & $5,8^{\mathrm{cd}}$ & $7,3^{\mathrm{ab}}$ & $6,5^{\mathrm{a}}$ \\
$\mathrm{D}_{\mathrm{F}}$ & 6,7 & $7,2^{\mathrm{ab}}$ & $6,0^{\mathrm{bcd}}$ & $5,6^{\mathrm{ab}}$ & $7,3^{\mathrm{a}}$
\end{tabular}

*Letras diferentes na mesma coluna indicam diferença significativa $(\mathrm{p} \leq 0,05)$ ** $\mathrm{F}=$ flan com acúcar; $\mathrm{P}=$ pudim com açúcar; $\mathrm{D}=$ pudim dietético. Letras maiúsculas em subscrito $(\mathrm{A}, \mathrm{B}, \mathrm{C}, \mathrm{D}, \mathrm{E}, \mathrm{F})$ indicam a marca dos produtos.

Uma outra parcela considerável $(32 \%, 18$ provadores do grupo 2) convergiu para a região inferior direita do mapa, onde se alocavam as amostras que apresentaram a terceira $\left(\mathrm{P}_{\mathrm{B}}\right)$ e quarta $\left(\mathrm{D}_{\mathrm{B}}\right)$ maiores médias. Interessante observar que nessa região, também estava alocado o $\mathrm{F}_{\mathrm{B}}$. (Figura 4). Esses consumidores apresentavam como característica em comum gostar da marca $\mathrm{B}$, independente da classe de produto (flan e pudim com açúcar e pudim dietético), e não apreciar outros pudins dietéticos (Tabela 4).

Em uma posição intermediária entre os grupos 1 e 2 , foram alocados os quatro consumidores identificados como grupo 3. Esses provadores gostavam dos produtos da marca B, mas se diferenciavam do grupo 2 por apreciar o $\mathrm{D}_{\mathrm{E}}$ (preferido pelo grupo 1) (Figura 4 e Tabela 4).

Na região à esquerda da Figura 4 estão localizados os consumidores (grupo 4) que rejeitavam o $\mathrm{D}_{\mathrm{E}}$ e/ou os produtos da marca $\mathrm{B}$ e apreciavam o $\mathrm{P}_{\mathrm{C}}$ e os produtos da marca A. Esse grupo representava uma minoria dos consumidores $(7 \%)$ que dava, no geral, notas mais altas às amostras e discriminava menos os produtos, mostrando comportamento oposto ao resto da equipe e atribuindo a maior nota $(8,3)$, a amostra que recebeu a menor nota média da equipe (Tabela 4). 
Os resultados mostram que, para essa equipe de consumidores, foi observada uma segmentação, e que cada grupo tinha seus critérios próprios de preferência. Dentre as amostras com maior porcentagem média de aprovação, o $\mathrm{P}_{\mathrm{B}}$ e $\mathrm{D}_{\mathrm{E}}$ foram efetivamente preferidos pelos provadores dos grupos 2 e 1 , respectivamente, e bem aceitos pelo grupo 3 . No entanto, o $\mathrm{D}_{\mathrm{A}}$, que apresentava a mesma nota média e porcentagem de aprovação, não foi o preferido de nenhum dos grupos.

Resultado similar havia sido relatado por DAMÁSIO, COSTELL \& DURAN [8] trabalhando com otimização da formulação de uma geléia de morango com baixo conteúdo de açúcar. A formulação selecionada de acordo com a preferência média de todo o grupo não foi a preferida em nenhum dos subgrupos. A identificação de grupos de consumidores com diferentes preferências e padrões de consumo permitiu a separação em dois grupos principais: um que gostava de geléias mais firmes, e outro que preferia géis com firmeza intermediária.

\subsection{2 - Componentes principais}

Para comparação com os resultados obtidos empregando-se a Escala Muldimensional, um Mapa de Preferência Interno foi gerado utilizando-se a Análise de Componentes Principais, que tem sido a técnica multivariada mais citada na literatura. A primeira e segunda dimensões explicaram $40 \%$ da variabilidade entre os indivíduos com relação à aceitação das diferentes amostras (Figura 5). Apesar da porcentagem de explicação obtida ser próxima à observada empregando-se EM, não foi observado resultado satisfatório.

Os dados de cada provador foram representados como um ponto no espaço, indivíduos com similaridades em uma ou mais propriedades (nesse caso, que tenham opinião semelhante sobre os produtos) estariam próximos (Figura 5a). As cargas ("loaddings") das variáveis (amostras) estão representadas na Figura $5 b$. Para facilitar a visualização, as amostras não estão na forma de vetores, mas representadas como pontos. A concentração de consumidores na região da amostra indicaria maior ou menor aceitação.
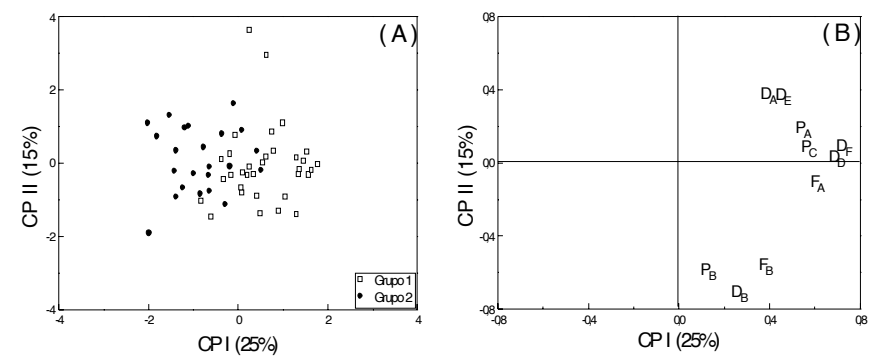

FIGURA 5. Projeção do parâmetro de aceitação para Componentes Principais I e II:

A) configuração dos provadores;

B) configuração das variáveis: $F=$ flan com açúcar; $\mathrm{P}=$ pudim com açúcar; $\mathrm{D}=$ pudim dietético. Letras maiúsculas em subscrito $(\mathrm{A}, \mathrm{B}, \mathrm{C}, \mathrm{D}, \mathrm{E}, \mathrm{F})$ indicam a marca dos produtos.
A primeira CP não permitiu discriminar as amostras com relação à preferência dos consumidores: produtos pouco apreciados $\left(\mathrm{P}_{\mathrm{C}}\right.$ e $\left.\mathrm{F}_{\mathrm{A}}\right)$ ficaram próximos a produtos mais apreciados $\left(\mathrm{D}_{\mathrm{E}}\right.$ e $\left.\mathrm{D}_{\mathrm{F}}\right)$ (Figura 5 b). Empregando-se a Análise de Agrupamentos observou-se no dendrograma a divisão em dois grupos principais de provadores com preferências diferenciadas. Graficamente, no entanto, não se observou visualmente a separação entre esses grupos (Figura $5 a$ ).

A Tabela 5 mostra as diferenças entre as notas médias recebidas pelas amostras nos grupos 1 (33 consumidores) e 2 (23 consumidores).

TABELA 5. Notas médias de aceitação das amostras considerando-se toda a equipe e cada grupo*.

\begin{tabular}{cccc}
\hline $\begin{array}{c}\text { Provadores/ } \\
\text { amostras }^{\star \star}\end{array}$ & Equipe & Grupo 1 & Grupo 2 \\
\hline $\mathrm{N}^{\circ}$ total & 56 & 33 & 23 \\
\hline $\mathrm{F}_{\mathrm{A}}$ & 5,8 & $6,2^{\mathrm{b}}$ & $5,1^{\mathrm{bc}}$ \\
$\mathrm{F}_{\mathrm{B}}$ & 6,5 & $6,8^{\mathrm{ab}}$ & $6,0^{\mathrm{ab}}$ \\
\hline $\mathrm{P}_{\mathrm{A}}$ & 6,1 & $6,6^{\mathrm{b}}$ & $5,4^{\mathrm{abc}}$ \\
$\mathrm{P}_{\mathrm{B}}$ & 6,9 & $6,9^{\mathrm{ab}}$ & $6,9^{\mathrm{a}}$ \\
$\mathrm{P}_{\mathrm{C}}$ & 5,5 & $6,2^{\mathrm{b}}$ & $4,5^{\mathrm{c}}$ \\
\hline $\mathrm{D}_{\mathrm{A}}$ & 6,9 & $7,1^{\mathrm{ab}}$ & $6,7^{\mathrm{a}}$ \\
$\mathrm{D}_{\mathrm{B}}$ & 6,8 & $7,2^{\mathrm{ab}}$ & $6,2^{\mathrm{ab}}$ \\
$\mathrm{D}_{\mathrm{D}}$ & 6,7 & $7,8^{\mathrm{a}}$ & $5,1^{\mathrm{bc}}$ \\
$\mathrm{D}_{\mathrm{E}}$ & 7,0 & $7,2^{\mathrm{ab}}$ & $6,7^{\mathrm{a}}$ \\
$\mathrm{D}_{\mathrm{F}}$ & 6,7 & $7,3^{\mathrm{ab}}$ & $5,8^{\mathrm{abc}}$ \\
\hline
\end{tabular}

*Letras diferentes na mesma coluna indicam diferença significativa $(\mathrm{p} \leq 0,05)$ ** $\mathrm{F}=$ flan com açúcar; $\mathrm{P}=$ pudim com açúcar; $\mathrm{D}=$ pudim dietético. Letras maiúsculas em subscrito (A, B, C, D, E, F) indicam a marca dos produtos.

Foram alocados mais à direita na Figura $5 a$ os consumidores (grupo 1) que, no geral, deram notas mais altas para todos os produtos e apreciavam os produtos dietéticos. Foram configurados mais à esquerda, os provadores (grupo 2) que diferenciavam mais entre os produtos e atribuíam, na média, notas mais baixas. Dessa forma, devido à pequena diferenciação de aceitação entre amostras, a maior parte da variabilidade da CP I parece ter sido empregada para explicar a maior ou menor aprovação aos produtos em geral (notas médias) e não, especificamente, preferência em relação às amostras.

Com relação ao segundo componente principal $\left(\mathrm{Fi}^{-}\right.$ gura $5 a$ ), observou-se que foi o responsável pela diferenciação da marca $\mathrm{B}$ em relação às outras. Observando-se as notas individuais, constatou-se que os provadores correlacionados negativamente com o CP II atribuíram maiores notas aos produtos da marca $\mathrm{B}$, independentemente da classe do produto (flan, pudim com açúcar ou dietético) e ficaram alocados na parte inferior do gráfico.

\section{4 - CONCLUSÕES}

Os produtos apresentaram, no geral, uma boa aceitação, independente de o consumidor estar acostumado a consumir produtos dietéticos e edulcorantes. Não 
foi observada diferença entre produtos com açúcar e dietéticos.

O emprego da técnica de Escala Muldimensional associada à Análise de Agrupamentos, proporcionou uma avaliação mais abrangente do comportamento dos provadores do que a Análise de Componentes Principais, permitindo identificar dois grandes grupos de consumidores: os que preferiam os produtos (pudins e flan) de uma determinada marca (B) e os que preferiam os pudins dietéticos.

A utilização de dados de aceitação média anulou os efeitos de segmentação do conjunto total de dados afetivos. Os resultados demonstram a eficiência do emprego do mapa para identificação e caracterização de preferências e grupos de consumidores.

\section{5 - REFERÊNCIAS BIBLIOGRÁFICAS}

[1] ALONSO, S.; SETSER, C. Functional replacements for sugars in foods. Trends in Food Science \& Technology, Cambridge, v. 5, n. 5, p. 139-146, 1994.

[2] ARDITTI, S. Preference mapping: a case study. Food Quality and Preference, Oxford, v. 8, n. 5/6, p. 323327, 1997.

[3] BEHRENS, J.H.; SILVA, M.A.A.P.; WAKELING, I.N. Avaliação da aceitação de vinhos brancos varietais brasileiros através de testes sensoriais afetivos e técnica multivariada de Mapa de preferência Interno. Ciênc. Tecnol. Aliment., Campinas, v. 19, n. 2, p. 214-220, 1999.

[4] CÂNDIDO, L.M.B.; CAMPOS, A.M. Alimentos para fins especiais: dietéticos. São Paulo, Livraria Varela. 1996. 423p.

[5] CARDELLO, H.M.A.B.; SILVA, M.A.A.P.; DAMÁSIO, M.H. Análise tempo-intensidade dos estímulos doce e amargo de extrato de folhas de estévia [ Stevia rebauduiana (Bert.) Bertoni] em doçura equivalente a sacarose. Ciênc. Tecnol. Aliment., Campinas, v. 19, n. 2, p. 163-169, 1999.

[6] CARNEIRO, J.C.S. Processamento Industrial de feijão, avaliação sensorial descritiva e mapa de preferência. Viçosa, 2001. 90p. Dissertação de Mestrado. Universidade Federal de Viçosa.

[7] CHAN, M.M.; MARTINELLI, K. The effect of color on perceived flavor intensity and acceptance of foods by young adults and elderly adults. Journal of the American Dietetic Association, Chicago, v. 56, n. 6, p. 657-659, 1997.

[8] DAMÁSIO, M.H.; COSTELL, E.; DURAN, L. Optimising acceptability of low-sugar strawberry gels segmenting consumers by internal preference mapping. Journal of the Science of Food and Agriculture, Sussex, v. 79, n. 4 , p. 626-632, 1999.

[9] ELMORE, J.R.; HEYMANN, H.; JOHNSON, J.; HEWETT,
J.E. Preference mapping: relating acceptance of "creaminess" to a descriptive sensory map of a semisolid. Food Quality and Preference, Oxford, v. 10, p. 465-475, 1999.

[10] FAS Foreign Agricultural Service - United States Department of Agriculture. Global Agricultural Information Network - Brasil. Disponivel em: www.fas.usda.gov/gainfiles/200203/135683610.pdf. Acesso em 23 de março de 2003.

[11] IOP, S.C.F.; BELEIA, A.P.; SILVA, R.S.F. Formulation and evaluation of dry dessert mix containing sweetener combinations using mixture response methodology. Food Chemistry, Oxford, v. 66, p. 167-171, 1999.

[12] LAWLESS, H.T.; HEYMANN, H. Sensory evaluation of food. New York: Chapman \& Hall, 1998. 819 p.

[13] MacFIE, H.J.H.; THOMSON, D.M.H. Preference mapping and multidimensional scaling. In: PIGGOTT, J.R. (Ed.). Sensory Analysis of Food. $2^{\circ}$ ed. New York, Elsevier. 1988. 389p.

[14] MacFIE, H.J.H. Assessment of the sensory properties of food. Nutrition Reviews, Washington, v. 48, n. 2, p. 87-93, 1990.

[15] MARKETTO, C.G.; COOPER, M.F.; PETTY, M.E.; SCRIVEN, F.M. The reliability of MDPREF to show individual preference. Journal of Sensory Studies, Trumbull, v. 3, n. 9, p. 337-350, 1994.

[16] OLIVEIRA, A.P.; FRASSON, K.; BENASSI, M.T. Pudins e flans de chocolate com açúcar e dietéticos: avaliação de cor e textura. In: CONGRESSO BRASILEIRO DE CIÊNCIA E TECNOLOGIA DE ALIMENTOS, 18., 2002, Porto Alegre. Anais.... Porto Alegre, p. 3541-3544

[17] OLIVEIRA, A.P.; FRASSON, K.; YAMASHITA, F.; BENASSI, M.T. Medida instrumental de cor em sobremesas lácteas de chocolate: uma técnica de baixo custo e versátil utilizando câmara digital. (submetido ao Brazilian Journal of Food Technology) 2002.

[18] OP \& P PRODUCT RESEARCH, Senstools Versão 2.3. Utrecht: OP \& P Product Research, 1995-1998. Conjunto de Programas 1CD room.

[19] SAS Institute, Versão 6.12. Cary: SAS Institute, 1989 1996.

[20] SCHLICH, P.; McEWAN, J.A. Preference mapping a statistical tool for the food industry. Science des Aliments, Paris, v. 12, p. 339-355, 1992.

[21] SMITH, S.M. PC-MDS Muldimensional Statistic Package. Institute of Business MGT-Brigham Young University, Provo, USA. 1990. Disponível em: www.marketing. byu.edu. Acesso em 23 março 2003.

[22] STATISTICA for Windows - Computer program manual. Tulsa: Statsoft, Inc., 1995.

[23] VIGNEAU, E.; QANNARI, E.M.; PUNTER, P.H.; KNOOPS, S. Segmentation of a panel of consumers using clustering of variables around latent directions of preference. Food Quality and Preference, Oxford, v. 12, p. 359-363, 2001. 